\title{
ERGODIC PROPERTIES OF BOGOLIUBOV AUTOMORPHISMS IN FREE PROBABILITY
}

\author{
FRANCESCO FIDALEO AND FARRUKH MUKHAMEDOV
}

\begin{abstract}
We show that some $C^{*}$-dynamical systems obtained by "quantizing" classical ones on the free Fock space, enjoy very strong ergodic properties. Namely, if the classical dynamical system $(X, T, \mu)$ is ergodic but not weakly mixing, then the resulting quantized system $(\mathfrak{G}, \alpha)$ is uniquely ergodic (w.r.t the fixed point algebra) but not uniquely weak mixing. The same happens if we quantize a classical system $(X, T, \mu)$ which is weakly mixing but not mixing. In this case, the quantized system is uniquely weak mixing but not uniquely mixing. Finally, a quantized system arising from a classical mixing dynamical system, will be uniquely mixing. In such a way, it is possible to exhibit uniquely weak mixing and uniquely mixing $C^{*}$-dynamical systems whose GNS representation associated to the unique invariant state generates a von Neuman factor of one of the following types: $I_{\infty}, I I_{1}, I I I_{\lambda}$ where $\lambda \in(0,1]$. The results listed above are extended to the $q$-commutation relations, provided $|q|<\sqrt{2}-1$.
\end{abstract}

Mathematics Subject Classification: 37A30, 46L55, 20E06.

Key words: unique ergodicity, mixing; Bogoliubov automorphism; $C^{*}$ dynamical systems; free probability.

\section{INTRODUCTION}

The study of quantum dynamical systems has been an impetuos growth in the last years, in view of natural applications to various field of mathematics and physics. It is then of interest to understand among the various ergodic properties, which ones survive and are meaningful, by passing from the classical to the quantum case. Due to noncommutativity, in the latter situation the matter is much more complicated than in the former. The reader is referred e.g. to $[3,17,27]$ for further details relative to some differences between classical and quantum situations. Therefore, it is then natural to study of the possible generalizations to quantum case of the various ergodic properties known for classical dynamical systems.

By coming back to the classical case, one of the strong ergodic properties of the dynamical system $(\Omega, T)$ consisting of a compact metric space $\Omega$ and a homeomorphism $T$, is the unique ergodicity which means that there exists a unique invariant Borel measure $\mu$ for $T$. It is known (cf. [21]) that the last property is equivalent to the uniform convergence in $C(\Omega)$ of the ergodic averages $\frac{1}{n} \sum_{k=0}^{n-1} f \circ T^{k}$ to $\int f \mathrm{~d} \mu$ for any $f \in C(\Omega)$. A pivotal example of 
classical uniquely ergodic dynamical system is given by an irrational rotation on the unit circle (see [9] for further examples). In the quantum setting, the last property is formulated as follows. Let $(\mathfrak{A}, \alpha)$ be a $C^{*}$-dynamical system consisting of the $C^{*}$-algebra $\mathfrak{A}$ and the automorphism $\alpha$. The unique ergodicity for $(\mathfrak{A}, \alpha)$ is equivalent (cf. $[1,2,25])$ to the norm convergence

$$
\lim _{n \rightarrow+\infty} \frac{1}{n} \sum_{k=0}^{n-1} \alpha^{n}(a)=E(a), \quad a \in \mathfrak{A} .
$$

Here, $E=\omega(\cdot) \mathbb{I}$ is the conditional expectation onto the fixed point subalgebra of $\alpha$ consisting of the constant multiples of the identity, and $\omega \in \mathcal{S}(\mathfrak{A})$ is the unique invariant state for $\alpha$, here $S(\mathfrak{A})$ denotes the set of all states of $\mathfrak{A}$. A natural generalization requires that the the fixed point subalgebra for $\alpha$ in (1.1) is nontrivial. This property, denoted as the unique ergodicity w.r.t. the fixed point subalgebra, has been investigated in $[1,2]$. The unique weak mixing was investigated in $[14,25]$, which means that

$$
\lim _{n \rightarrow+\infty} \frac{1}{n} \sum_{k=0}^{n-1}\left|\varphi\left(\alpha^{k}(a)\right)-\varphi(E(a))\right|=0, \quad a \in \mathfrak{A},
$$

for every $\varphi \in \mathcal{S}(\mathfrak{A})$. As before, $E$ is the unique conditional expectation projecting onto the fixed point subalgebra. Finally, the unique mixing was defined and investigated in [13]. We simply require that

$$
\lim _{n} \varphi\left(\alpha^{n}(a)\right)=\varphi(E(a)), \quad a \in \mathfrak{A},
$$

for every $\varphi \in \mathcal{S}(\mathfrak{A})$.

The property (1.2) of the convergence to the equilibrium is perfectly meaningful in the quantum setting but its classical counterpart is the following: if a classical system fulfils (1.2) with $E(f)=\int f \mathrm{~d} \mu$, the support of the unique invariant measure $\mu$ is a singleton, that is, it is conjugate to the trivial one point dynamical system, see [13]. Indeed, we can exhibit some interesting examples of uniquely mixing $C^{*}$-dynamical system in the quantum setting, for which the fixed point algebra is trivial or non trivial as well. Such examples are constructed by quantizing the shift on $\ell^{2}(\mathbb{Z})$ on the Boltzmann-Fock space $\mathcal{F}\left(\ell^{2}(\mathbb{Z})\right)$. Other examples arise from some generalizations of the free shift to the shift on the reduced $C^{*}$-algebras of $R D$-groups, and the free amalgamated product of $C^{*}$-algebras (cf. [13, 14]), respectively. Among these case, there are examples of dynamical systems which are uniquely mixing w.r.t. the fixed point algebra (i.e. for which there are many invariant states, see below) as well. Finally, in [11] it has been established that the shift automorphism of the $q$-deformed Canonical Commutation Relations algebra enjoys unique mixing property. We can then exhibit uniquely mixing $C^{*}$-dynamical system for which the von Neumman algebra generated by the GNS representation of the unique invariant state is a type $I_{\infty}$ factor (case of the unital algebra $\mathfrak{R}$ acting on the Boltzmann-Fock space $\mathcal{F}(\mathcal{H})$ generated by the annihilators $a(f), f \in \mathcal{H}$ ), or a type $I I_{1}$ factor (case of the unital algebra $\mathfrak{G}$ acting on the 
Boltzmann-Fock space generated by the selfadjoint part of the annihilators $\left.a(f)+a^{+}(f), f \in \mathcal{H}\right)$. The reader is referred to the papers $[1,2,11,13,14,24]$ for further details on the topic. It is then natural to address the possibility to exhibit further examples of $C^{*}$-dynamical systems enjoying all the ergodic properties such that the von Neumann algebra generated by the GNS representation of the unique invariant state is a factor of different type from the previous ones. The aim of the present paper is to show that this is indeed possible by quantizing classical dynamical systems satisfying the corresponding ergodic properties.

Let $\mathcal{H}$ be a separable Hilbert space. Consider for $-1 \leq q \leq 1$ the $q$-canonical commutation relations for annihilators $a(f)$ and creators $a^{+}(f)$ :

$$
a(f) a^{+}(g)-q a^{+}(g) a(f)=\langle g, f\rangle_{\mathcal{H}} \mathbb{I}, \quad f, g \in \mathcal{H} .
$$

The case $q=-1$ is the Fermionic (Canonical Anticommutation Relation) case, whereas the case $q=1$ is the Bosonic (Canonical Commutation Relation) case, and finally $q=0$ corresponds to the Boltzmann (or free) case. For unitaries $U$ acting on $\mathcal{H}$, the Bogoliubov automorphisms, defined as $\alpha_{U}(a(f)):=a(U f)$ are widely investigated (e.g. [6]) in the CAR and CCR cases for the natural physical applications. The reader is referred to [4, 8, 10, 26, 29], for various results and applications, including the computation of various kind of entropies of the Bogoliubov automorphisms. In [7] (see also [32]) it has been pointed out by a few words, that the free shifts of the Cuntz algebra $\mathcal{O}_{\infty}$ and on $C_{r}^{*}\left(\mathbb{F}_{\infty}\right)$ are "highly ergodic". In the present paper (see also the previous one [13]) the meaning of the previuos sentence is then clarified: the Bogoliubov automorphisms, including the shift, are highly ergodic automorphisms as they enjoy one of the strong ergodic properties of unique ergodicity, unique weak mixing or unique mixing described below.

We start from a classical dynamical system $(X, T, \mu)$ consisting of a probability space $(X, \mu)$, and a measure preserving invertible transformation $T$ : $X \mapsto X$. By using the Shlyakhtenko construction on the Boltzmann-Fock space (cf. $[28,16]$ ), we consider the Bogoliubov automorphism $\alpha_{U}$ relative to the unitary $U$ associated to the measure preserving transformation $T$. We obtain the following results. If the classical dynamical system $(X, T, \mu)$ is ergodic but not weakly mixing, then the resulting quantized system $(\mathfrak{G}, \alpha)$ is uniquely ergodic w.r.t. the fixed point algebra (which is always nontrivial in this situation), but not uniquely weak mixing. If we quantize a classical system $(X, T, \mu)$ which is weakly mixing but not mixing, the resulting quantized system is uniquely weak mixing but not uniquely mixing. Finally, if we quantize a mixing system $(X, T, \mu)$, the resulting quantum system will be uniquely mixing. In such a way, it is possible to exhibit uniquely weak mixing and uniquely mixing $C^{*}$-dynamical systems whose von Neumann algebra generated by the GNS representation associated to the unique invariant state is a factor of type $I_{\infty}, I I_{1}$ or $I I I_{\lambda}$ where $\lambda \in(0,1]$.

For the sake of completeness, our results are extended to the $C^{*}$-dynamical systems based on the $q$-commutation relations, provided $|q|<\sqrt{2}-1$. 
To end the present introduction, we point out few things. At the classical level we have a wide class of uniquely ergodic dynamical systems. The reader is referred to [9] and the literature cited therein. In addition, starting from a measure preserving ergodic dynamical system $(X, T, \mu)$, it is possible to construct in a canonical way a uniquely ergodic classical dynamical system $\left(C(Y), \alpha_{S}\right)$ such that $(Y, S, \nu)$ is conjugate to $(X, T, \mu), \nu$ being the unique invariant probability measure on $Y$ invariant under $S .^{1}$ This is nothing but the Jewett-Krieger Theorem (cf. [18, 22]). For the intermediate weak mixing situation nothing is yet known and the Jewett-Krieger Theorem is not yet available in this case. Finally, for the mixing case, any classical dynamical system enjoying $(1.2)$ with $E=\omega(\cdot) \mathbb{I}$ is conjugate to the one point trivial dynamical system and then the Jewett-Krieger Theorem cannot be carry out. Notice that our approach is similar to the Jewett and Krieger one at least in principle. Namely, starting from a classical dynamical system based on an measure preserving transformation which is ergodic, weakly mixing or mixing, we can construct in a functorial way, nontrivial quantum dynamical systems (one for each type $I_{\infty}, I I_{1}$ or $I I I_{\lambda}, \lambda \in(0,1]$ of von Neumann factor) enjoying the unique ergodicity, unique weak mixing or unique mixing, respectively.

\section{PRELIMINARIES}

In this section we recall some preliminaries concerning $C^{*}$-dynamical systems.

Let $\mathfrak{A}$ be a $C^{*}$-algebra with unit $\mathbb{I}$. By $\mathcal{S}(\mathfrak{A})$ we denote the set of all states on $\mathfrak{A}$. For a (discrete) $C^{*}$-dynamical system we mean a triplet $(\mathfrak{A}, \alpha, \omega)$ consisting of a unital $C^{*}$-algebra $\mathfrak{A}$, an automorphism $\alpha$ of $\mathfrak{A}$, and a state $\omega \in \mathcal{S}(\mathfrak{A})$ invariant under the action of $\alpha$. The pair $(\mathfrak{A}, \alpha)$ consisting of a unital $C^{*}$-algebra and an automorphism as before is called a $C^{*}$-dynamical system as well. Suppose now that the classical dynamical system $(X, T, \mu)$ is merely based of a probability space $(X, \mu)$, and a measure preserving invertible transformation $T: X \mapsto X$. It is well known that $T$ induces a unitary transformation acting on $L^{2}(X, \mu)$. Consider the natural restriction of $U$ to $\mathcal{H}:=L^{2}(X, \mu) \ominus \mathbb{C} 1$, being 1 the constant function. It is known (see e.g. [21]) that the dynamical system $(X, T, \mu)$ is ergodic, weak mixing, mixing iff

$$
\begin{gathered}
\lim _{n \rightarrow \infty} \frac{1}{n} \sum_{k=1}^{n}\left\langle U^{k} \xi, \eta\right\rangle=0, \quad \xi, \eta \in \mathcal{H}, \\
\lim _{n \rightarrow \infty} \frac{1}{n} \sum_{k=1}^{n}\left|\left\langle U^{k} \xi, \eta\right\rangle\right|=0, \quad \xi, \eta \in \mathcal{H}, \\
\lim _{n \rightarrow \infty}\left\langle U^{n} \xi, \eta\right\rangle=0, \quad \xi, \eta \in \mathcal{H},
\end{gathered}
$$

\footnotetext{
${ }^{1}$ The measure preserving transformations $\left(X_{j}, T_{j}, \mu_{j}\right), j=1,2$, are said to be conjugate if there exist $\mu_{j}$-measurable sets $A_{j} \in X_{j}$ of full measure such that $T_{j}\left(A_{j}\right)=A_{j}$, and a one-to-one measure preserving map $S: A_{1} \mapsto A_{2}$ such that $T_{2}=S \circ T_{1} \circ S^{-1}$.
} 
respectively. In the present paper, we call with an abuse of notations, any unitary $U$ acting on a Hilbert space $\mathcal{H}$ satisfying (2.1), (2.2), (2.3), ergodic, weakly mixing, or mixing, respectively.

Let $(\mathfrak{A}, \alpha)$ be a $C^{*}$-dynamical system, and $E: \mathfrak{A} \mapsto \mathfrak{A}$ a linear map. suppose that

$$
\begin{gathered}
\lim _{n \rightarrow+\infty} \frac{1}{n} \sum_{k=0}^{n-1} \varphi\left(\alpha^{k}(x)\right)=\varphi(E(x)), \quad x \in \mathfrak{A}, \varphi \in \mathcal{S}(\mathfrak{A}), \\
\lim _{n \rightarrow+\infty} \frac{1}{n} \sum_{k=0}^{n-1}\left|\varphi\left(\alpha^{k}(x)\right)-\varphi(E(x))\right|=0, \quad x \in \mathfrak{A}, \varphi \in \mathcal{S}(\mathfrak{A}),
\end{gathered}
$$

or finally,

$$
\lim _{n \rightarrow+\infty} \varphi\left(\alpha^{n}(x)\right)=\varphi(E(x)), \quad x \in \mathfrak{A}, \varphi \in \mathcal{S}(\mathfrak{A}) .
$$

It can readily seen (cf. [14]) that the map $E$ is a conditional expectation projecting onto the fixed point subalgebra $\mathfrak{A}^{\alpha}:=\{x \in \mathfrak{A}: \alpha(x)=x\}$. Furthermore, $E$ is invariant w.r.t. $\alpha$.

Definition 2.1. ( $\mathfrak{A}, \alpha)$ is said to be uniquely ergodic, uniquely weak mixing or uniquely mixing w.r.t the fixed point subalgebra, if (2.4), (2.5) or (2.6) holds true, respectively.

If $E=\omega(\cdot) \mathbb{I}$, then we simply call the dynamical system $(\mathfrak{A}, \alpha)$ uniquely ergodic, uniquely weak mixing or uniquely mixing (UE, UWM and UM for short), respectively. ${ }^{2}$

By using the Jordan decomposition of bounded linear functionals, one can replace $\mathcal{S}(\mathfrak{A})$ with $\mathfrak{A}^{*}$ everywhere in Definition 2.1. We refer to [30, 31] for standard results on the operator algebras and modular theory.

Let $\mathcal{H}$ be a separable Hilbert space. The Boltzmann-Fock space (called sometimes full Fock space) $\mathcal{F}(\mathcal{H})$ is defined by

$$
\mathcal{F}(\mathcal{H}):=\mathbb{C} \Omega \oplus_{n=1}^{\infty} \mathcal{H}^{\otimes n} .
$$

The vector $\Omega$ is called the vacuum vector, and the vector state $\omega:=\langle\cdot \Omega, \Omega\rangle$ the vacuum state For $f \in \mathcal{H}$, the (left) creator $a^{+}(f)$ acts on $\mathcal{F}(\mathcal{H})$ by

$$
a^{+}(f) \Omega=f, \quad a^{+}(f) f_{1} \otimes \cdots \otimes f_{n}=f \otimes f_{1} \otimes \cdots \otimes f_{n},
$$

and its adjoint is the (left) annihilator $a(f)$ given by

$$
a(f) \Omega=0, \quad a(f) f_{1} \otimes \cdots \otimes f_{n}=\left\langle f_{1}, f\right\rangle f_{2} \otimes \cdots \otimes f_{n} .
$$

It is easily seen that $a(f)^{*}=a^{+}(f)$, and the $a(f)$ satisfy the commutation rule (1.3) with $q=0$. Let $P: \mathcal{H} \mapsto \mathcal{H}$ be a positive contraction. Then the map $\mathcal{F}(P): \mathcal{F}(\mathcal{H}) \mapsto \mathcal{F}(\mathcal{H})$ defined as

$$
\mathcal{F}(P) \Omega:=\Omega, \quad \mathcal{F}(P) f_{1} \otimes \cdots \otimes f_{n}:=P f_{1} \otimes \cdots \otimes P f_{n}
$$

\footnotetext{
${ }^{2}$ If $E=\omega(\cdot) \mathbb{I}$, then there is a unique invariant state for $\alpha$, see [1].
} 
is a contraction. The induced map $T_{P}(a(f))=a(P f)$ is a completely positive map on the unital $C^{*}$-algebra generated by the all the annihilators. If $U$ is an isometry (resp. unitary), then $T_{U}$ is an endomorphism (resp. automorphism). In the sequel we are interested in the case when $U$ is unitary. Then $T_{U}$ is called a Bogoliubov automorphism (see [16, 28, 33]). Note that dynamical and topological entropies of such kind of automorphisms were intensively studied in $[4,15,29,32]$ for the CAR and the CCR cases. The unique case relative to $q=0$ concerns the quantization of the shift on $\ell^{2}(\mathbb{Z})$, see e.g $[7,8]$. Concerning the entropy, nothing is yet known for a general Bogoliubov automorphism in the case $-1<q<1$.

\section{ERGODIC PROPERTIES OF BOGOLIUbOV AUTOMORPHISMS}

In the present paper we assume that all the Hilbert spaces we deal with are separable even if it is not directly specified.

Let $\mathcal{H}$ be a separable Hilbert space and $U: \mathcal{H} \rightarrow \mathcal{H}$ be an unitary. By $\alpha_{U}$ we denote the Bogoliubov automorphism $T_{U}$. We start with the the following estimation needed in the sequel.

Proposition 3.1. Let $\left\{k_{l}\right\}_{l \in \mathbb{N}}$ be any subsequence of natural numbers, and $U$ be a unitary operator. Under the above notations, we have the following estimation

$$
\begin{aligned}
& \left\|\sum_{l=1}^{N} \alpha_{U}^{k_{l}}\left(a^{+}\left(f_{1}\right) \cdots a^{+}\left(f_{m}\right) a\left(g_{1}\right) \cdots a\left(g_{n}\right)\right)\right\| \\
\leq & \left.\| \sum_{l=1}^{N} U^{-k_{l}} f_{1} \otimes \cdots \otimes U^{-k_{l}} f_{m} \otimes U^{k_{l}} g_{n} \otimes \cdots \otimes U^{k_{l}} g_{1}\right) \| .
\end{aligned}
$$

Proof. It is enough to consider $x \in \mathcal{H}^{\otimes t}$ with $t \geq n$. By using any orthonormal basis $\left\{e_{j}\right\}_{j \in J}$ for $\mathcal{H}$, we can symbolically write

$$
x=\sum_{\sigma_{1}, \ldots, \sigma_{n}, \mathbf{s}} x_{\sigma_{1}, \ldots, \sigma_{n}, \mathbf{s}} e_{\sigma_{1}} \otimes \cdots \otimes e_{\sigma_{n}} \otimes \xi_{\mathbf{s}}
$$

with $\left\langle\xi_{\mathbf{r}}, \xi_{\mathbf{s}}\right\rangle=\delta_{\mathbf{r s}}$. We can also suppose that the $x_{\sigma_{1}, \ldots, \sigma_{n}, \mathbf{s}}$ are zero but finitely many of them. Put $F:=f_{1} \otimes \cdots \otimes f_{m}, G:=g_{n} \otimes \cdots \otimes g_{1}$. We have

$$
\begin{gathered}
\Gamma:=\sum_{l=1}^{N} \alpha_{U}^{k_{l}}\left(a^{+}\left(f_{1}\right) \cdots a^{+}\left(f_{m}\right) a\left(g_{1}\right) \cdots a\left(g_{n}\right)\right) x \\
=\sum_{l=1}^{N} \sum_{\mathbf{s}}\left\langle\sum_{\sigma_{1}, \ldots, \sigma_{n}} x_{\sigma_{1}, \ldots, \sigma_{n}, \mathbf{s}} e_{\sigma_{1}} \otimes \cdots \otimes e_{\sigma_{n}},\left(U^{\otimes n}\right)^{k_{l}} G\right\rangle\left(U^{\otimes m}\right)^{k_{l}} F \otimes \xi_{\mathbf{s}} .
\end{gathered}
$$


It follows that $\langle\Gamma, \Gamma\rangle$ can be viewed as a linear combinations of inner products in $\mathcal{H}^{\otimes(2 n+m)}$, obtaining

$$
\begin{aligned}
& \langle\Gamma, \Gamma\rangle=\sum_{\mathbf{s}}\left\langle\left(\sum_{\sigma_{1}, \ldots, \sigma_{n}} x_{\sigma_{1}, \ldots, \sigma_{n}, \mathbf{s}} e_{\sigma_{1}} \otimes \cdots \otimes e_{\sigma_{n}}\right)\right. \\
& \otimes\left(\sum_{l=1}^{N}\left(U^{\otimes m}\right)^{-k_{l}} F \otimes\left(U^{\otimes n}\right)^{k_{l}} G\right),\left(\sum_{l=1}^{N}\left(U^{\otimes n}\right)^{k_{l}} G \otimes\left(U^{\otimes m}\right)^{-k_{l}} F\right) \\
& \left.\otimes\left(\sum_{\sigma_{1}, \ldots, \sigma_{n}} x_{\sigma_{1}, \ldots, \sigma_{n}, \mathbf{s}} e_{\sigma_{1}} \otimes \cdots \otimes e_{\sigma_{n}}\right)\right\rangle \\
& \leq\left\|\sum_{l=1}^{N}\left(U^{\otimes m}\right)^{-k_{l}} \otimes\left(U^{\otimes n}\right)^{k_{l}} F \otimes G\right\|^{2} \| \sum_{\mathbf{s}} x_{\sigma_{1}, \ldots, \sigma_{n}} \\
& =\| \sum_{l=1}^{N}\left(U^{\otimes m}\right)^{-k_{l}, \mathbf{s}} \otimes\left(U_{\sigma_{1}}^{\otimes n} \otimes \cdots \otimes e_{\sigma_{n}}\left\|^{2}\right\|^{k_{l}} F \otimes G \|_{\|x\|^{2} .}^{2}\right.
\end{aligned}
$$

Let $\mathcal{H}_{\mathbb{R}}$ be a separable real Hilbert space, and $U_{\mathbb{R}}: \mathcal{H}_{\mathbb{R}} \mapsto \mathcal{H}_{\mathbb{R}}$ be an orthogonal transformation. Extend $U_{\mathbb{R}}$ by linearity to $\mathcal{H}_{\mathbb{C}}:=\mathcal{H}_{\mathbb{R}}+i \mathcal{H}_{\mathbb{R}}$ and denote such a unitary operator as $U_{\mathbb{C}}$. We report the following known fact for the convenience of the reader.

Lemma 3.2. If $\sigma_{\mathrm{pp}}\left(U_{\mathbb{C}}\right) \neq \emptyset$, then $U_{\mathbb{R}} \otimes U_{\mathbb{R}}$ has a nontrivial invariant vector. Proof. Let $e^{i \theta} \in \sigma_{\mathrm{pp}}\left(U_{\mathbb{C}}\right)$ with eigenvector $v=x+i y$, with $x, y \in \mathcal{H}_{\mathbb{R}}$. Then

$$
U_{\mathbb{R}}\left(\begin{array}{l}
x \\
y
\end{array}\right)=\left(\begin{array}{cc}
\cos \theta & -\sin \theta \\
\sin \theta & \cos \theta
\end{array}\right)\left(\begin{array}{l}
x \\
y
\end{array}\right) .
$$

The vector we are searching for is nothing but $x \otimes x+y \otimes y .^{3}$

Now consider a real subspace $\mathcal{K} \subset \mathcal{H}$ of the Hilbert space $\mathcal{H}$ and suppose that the unitary operator $U$ acting on $\mathcal{H}$ satisfies $U \mathcal{K} \subset \mathcal{K}, U^{*} \mathcal{K} \subset \mathcal{K}{ }^{4}$ Let $\left(\mathfrak{R}_{\mathcal{K}}, \alpha_{U}\right)$ be the $C^{*}$-dynamical system, where $\mathfrak{R}_{\mathcal{K}}$ is the unital $C^{*}$-algebra acting on $\mathcal{F}(\mathcal{H})$ generated by $\{a(f) \mid f \in \mathcal{K}\}$, and $\alpha_{U}$ the restriction of $T_{U}$ to $\mathfrak{R}_{\mathcal{K}}$. The $C^{*}$-dynamical system $\left(\mathfrak{G}_{\mathcal{K}}, \alpha_{U}\right)$ consists of the unital $C^{*}$-algebra acting on $\mathcal{F}(\mathcal{H})$ generated by $\left\{s(f):=a(f)+a(f)^{+} \mid f \in \mathcal{K}\right\}$ and the restriction of $T_{U}$ to $\mathfrak{G}_{\mathcal{K}}$. Note that this is a Voiculescu's $C^{*}$-Gaussian functor, see [33]. In the sequel we simply write $(\mathfrak{R}, \alpha)$ and $(\mathfrak{G}, \alpha)$, respectively.

Proposition 3.3. If $U$ on $\mathcal{K}$ is ergodic, then the dynamical systems $(\mathfrak{R}, \alpha)$, $(\mathfrak{G}, \alpha)$ are ergodic w.r.t. the fixed point algebra.

\footnotetext{
${ }^{3}$ It follows that, if $e^{i \theta}= \pm 1$, then $U_{\mathbb{R}}$ has an eigenvector corresponding to $e^{i \theta}$. If $e^{i \theta} \in$ $\mathbb{T} \backslash\{ \pm 1\}$, then $U_{\mathbb{R}}$ has $\left(\begin{array}{cc}\cos \theta & -\sin \theta \\ \sin \theta & \cos \theta\end{array}\right)$ as a direct summand.

${ }^{4}$ In this case, $U\lceil\mathcal{K}$ defines an orthogonal transformation on $\mathcal{K}$, when the last is equipped with the inner product $(x, y):=\operatorname{Re}\langle x, y\rangle$.
} 
Proof. By a standard approximation argument, it is enough to consider the case when

$$
A:=a^{+}\left(f_{1}\right) \cdots a^{+}\left(f_{m}\right) a\left(g_{1}\right) \cdots a\left(g_{n}\right),
$$

where the $f_{1}, \ldots, f_{m}, g_{1} \ldots, g_{n}$ are either eigenvectors of $U$, or belong to the Hilbert subspace relative to the continuous spectrum of $U$. If $f_{1}, \ldots, f_{m}, g_{1} \ldots, g_{n}$ are eigenvectors of $U$ with corresponding eigenvalues $e^{i \theta_{1}}, \ldots, e^{i \theta_{m}}, e^{i \Theta_{1}} \ldots, e^{i \Theta_{n}}$ such that

$$
\sum_{i=1}^{m} \theta_{i}-\sum_{i=1}^{n} \Theta_{i}=2 h \pi
$$

for some integer $h \in \mathbb{Z}$, we conclude that $A$ is invariant under $\alpha$. Otherwise, the vector $f_{1} \otimes \cdots \otimes f_{m} \otimes g_{1} \otimes \cdots \otimes g_{n} \in \mathcal{H}^{\otimes(m+n)}$ is not invariant for the unitary $\left(U^{*}\right)^{\otimes m} \otimes U^{\otimes n}$. By Proposition 3.1 and the Mean Ergodic Theorem, we get in the last case $\lim _{N} \frac{1}{N} \sum_{k=1}^{N} \alpha^{k}(A)=0$, and the proof follows.

Proposition 3.4. If $U$ on $\mathcal{K}$ is weakly mixing (resp mixing), then the dynamical systems $(\mathfrak{R}, \alpha),(\mathfrak{G}, \alpha)$ are $U W M$ (resp. UM) with the vacuum state the unique invariant state under $\alpha$.

Proof. Let $A$ be as in (3.1). We have for any subsequence $\left\{k_{l}\right\}_{l \in \mathbb{N}}$ of natural numbers of positive lower density, $\lim _{N} \frac{1}{N} \sum_{l=1}^{N} \alpha^{k_{l}}(A)=0$ by taking into account Proposition 3.1 and the fact that $U$ (and then $\left(U^{*}\right)^{\otimes m} \otimes U^{\otimes n}$ ) is weakly mixing (cf. [19]). Again by [19], this implies that, for each $X \in \mathfrak{R}$ such that $\omega(X)=0$, the sequence $\left\{\alpha^{n}(X)\right\}_{n \in \mathbb{N}}$ is (uniformly) weakly mixing at 0 . It turns out to be equivalent to the fact that $(\mathfrak{R}, \alpha)$ is $\mathrm{UWM}$ with $\omega$ the unique invariant state. In the mixing case, by Proposition 3.1, we have for operators $A$ as before and any subsequence $\left\{k_{l}\right\}_{l \in \mathbb{N}}$ of natural numbers, $\lim _{N} \frac{1}{N} \sum_{l=1}^{N} \alpha^{k_{l}}(A)=0$ (cf. [23]). The proof follows by Proposition 2.3 of $[13]$.

Now we show that the quantized systems arising from ergodic but not weakly mixing classical dynamical systems, cannot be UWM w.r.t. the fixed algebra. The same will happen in the weak mixing situation: the resulting quantum system cannot be UM.

Proposition 3.5. Let $U$ be ergodic (resp weakly mixing) and suppose that there exists some $f \in \mathcal{K}$ such that the sequence $\left\{U^{k} f\right\}_{k \in \mathbb{N}}$ is not weakly mixing (resp. mixing) at 0 . Then the dynamical systems $(\mathfrak{R}, \alpha),(\mathfrak{G}, \alpha)$ cannot be UWM (resp UM) w.r.t. the fixed point algebra.

Proof. Let $f \in \mathcal{K}$ such that $\left\{U^{k} f\right\}_{k \in \mathbb{N}}$ is not weakly mixing (resp. mixing) at 0. By Proposition 3.3, the dynamical systems $(\mathfrak{R}, \alpha),(\mathfrak{G}, \alpha)$ are UE w.r.t. the fixed point algebra. Thus,

$$
\lim _{N} \frac{1}{N} \sum_{k=1}^{N} \alpha^{k}\left(a^{+}(f)\right)=E\left(a^{+}(f)\right)=0=E\lceil\mathfrak{G}(s(f)),
$$


being $E$ the conditional projection onto $\mathfrak{R}^{\alpha}$. According to [19] ([23]) there exists a subsequence $\left\{k_{l}\right\}_{l \in \mathbb{N}}$ of natural numbers of positive lower density (resp. a subsequence of natural numbers) such that

$$
\underset{N}{\limsup }\left\|\frac{1}{N} \sum_{l=1}^{N} U^{k_{l}} f\right\|>0 .
$$

Suppose that $(\mathfrak{R}, \alpha)$ or $(\mathfrak{G}, \alpha)$ is UWM (resp. UM) w.r.t. the fixed point algebra. Then one gets

$$
\begin{gathered}
0=\lim _{N} \frac{1}{N}\left\|\sum_{k=1}^{N} \alpha^{k}(s(f)) \Omega\right\|=\lim _{N}\left\|\frac{1}{N} \sum_{k=1}^{N} \alpha^{k}\left(a^{+}(f)\right) \Omega\right\| \\
=\limsup _{N}\left\|\frac{1}{N} \sum_{l=1}^{N} U^{k_{l}} f\right\|>0
\end{gathered}
$$

which is a contradiction.

\section{ON THE TYPE OF THE FACTORS GENERATED BY BOgOLIUBOV AUTOMORPHISMS}

In the present section we construct $C^{*}$-dynamical systems enjoining the strong ergodic properties listed in Section 2, and whose GNS representation relative to the Fock vacuum (which is the unique invariant state for the discrete dynamics in the case of UWM and UM) generates type $I I_{1}$ and type $I I I_{\lambda}$, $\lambda \in(0,1]$ von Neumann factors. This is done by quantizing any classical ergodic, weakly mixing or mixing dynamical system on the Boltzmann-Fock space.

Let $(X, T, \mu)$ be a classical dynamical system made of a probability space $(X, \mu)$, and a measure preserving invertible transformation $T: X \mapsto X$. We suppose that $L^{2}(X, \mu)$ is infinite dimensional. Let

$$
\mathcal{K}_{\mathbb{R}}:=\left(L_{\mathbb{R}}^{2}(X, \mu) \ominus \mathbb{R} 1\right) \bigotimes\left(\oplus_{\lambda \in G} \mathbb{R}^{2}\right) .
$$

Here, $1 \in L_{\mathbb{R}}^{2}(X, \mu)$ is the constant $\mathrm{f}$ unction which is invariant under the action of $U$, and $G$ is any countable multiplicative subgroup of $\mathbb{R}_{+}$. Let $u f:=f \circ T^{-1}$ and

$$
v(t):=\bigoplus_{\lambda \in G}\left(\begin{array}{cc}
\cos (t \ln \lambda) & -\sin (t \ln \lambda) \\
\sin (t \ln \lambda) & \cos (t \ln \lambda)
\end{array}\right) .
$$

Then $u \otimes I$ and $I \otimes v(t)$ are orthogonal transformations acting on the real Hilbert space $\mathcal{K}_{\mathbb{R}}$ satisfying $[u \otimes I, I \otimes v(t)]=0$. Let $\mathcal{K}_{\mathbb{C}}$ be the complexification of $\mathcal{K}_{\mathbb{R}}$ together with the positive non singular generator $A$ of the complexification of $I \otimes v(t)$ as $I \otimes v(t)=I \otimes a^{i t}=: A^{i t}$. Let $\mathcal{H}$ be the completion of $\mathcal{K}_{\mathbb{C}}$ with respect the inner product induced by $A$

$$
\langle x, y\rangle:=\left(2 A(I+A)^{-1} x, y\right),
$$

where $(\cdot, \cdot)$ is the inner product of $\mathcal{K}_{\mathbb{C}}$. Denote by $U$ and $V(t)$ the unitary extension of the corresponding orthogonal operators to the whole $\mathcal{H}$. Let $\mathcal{F}(\mathcal{H})$ 
be the full Fock space generated by $\mathcal{H}$ together with the Fock vacuum vector $\Omega$, and $\mathfrak{G}$ the $C^{*}$-algebra acting on $\mathcal{F}(\mathcal{H})$, generated by $\left\{s(f):=a(f)+a^{+}(f)\right.$ : $f \in \mathcal{K}\}$. Notice that $\Omega$ is cyclic for $\mathfrak{G}$ and $\mathfrak{G}^{\prime}$ (cf. [28]), that is $\Omega$ is a standard vector for $\mathfrak{G}^{\prime \prime}$. The dynamical system under consideration is $(\mathfrak{G}, \alpha)$, where $\alpha$ is the automorphism on $\mathfrak{G}$ induced by $\alpha(s(f)):=s(U f)$.

Proposition 4.1. If $(X, T, \mu)$ is ergodic but not weakly mixing, then the fixed point algebra $\mathfrak{G}^{\alpha}$ is nontrivial.

Proof. As $u$ is nontrivial and not weakly mixing, $U$ has at least an eigenvalue $\chi$ in $\mathbb{T} \backslash\{1\}$. If $\chi=-1$ there is a corresponding eigenvector $f \in \mathcal{K}$. An invariant element under the action of $\alpha$ is $s(f)^{2}$. If $\xi \in \mathbb{T} \backslash\{ \pm 1\}$, with the corresponding eigenvector $v=f+i g$, then by Lemma 3.2, an invariant element is given by $s(f)^{2}+s(g)^{2}$.

The main results of the present paper are summarized in the following theorems.

Theorem 4.2. Let $\alpha$ be the automorphism in $\mathfrak{G}$ induced by $\alpha(s(f)):=s(U f)$. Then the following assertions hold true.

(i) If $(X, T, \mu)$ is ergodic but not weakly mixing, then $(\mathfrak{G}, \alpha)$ is UE w.r.t $\mathfrak{G}^{\alpha}$, which is always nontrivial.

(ii) If $(X, T, \mu)$ is weakly mixing but not mixing, then $(\mathfrak{G}, \alpha)$ is $U W M$ but not $U M$, with $\omega$ as the unique invariant state.

(iii) If $(X, T, \mu)$ is mixing, then $(\mathfrak{G}, \alpha)$ is $U M$, with $\omega$ the unique invariant state.

Proof. We start by noticing that if $U$ is ergodic (resp. weakly mixing or mixing) in $\mathcal{K}_{\mathbb{C}}$, then its extension on $\mathcal{H}$ is ergodic (resp. weakly mixing or mixing) as well. This easily follows by (4.2) as, for any subsequence $\left\{k_{l}\right\}_{l \in \mathbb{N}}$ of natural number, we get

$$
\left\|\frac{1}{N} \sum_{l=1}^{N} U^{k_{l}} f\right\|_{\mathcal{H}} \leq\|A\|_{\mathcal{B}\left(\mathcal{K}_{\mathbb{C}}\right)}^{1 / 2}\left\|\frac{1}{N} \sum_{k=1}^{N} U^{k} f\right\|_{\mathcal{K}_{\mathbb{C}}},
$$

$A$ being the positive operator in (4.2). Then by Proposition 3.3 (resp. Proposition 3.4), $(\mathfrak{G}, \alpha)$ is UE w.r.t. the fixed point algebra (resp. UWM or UM with the Fock vacuum $\omega$ as the unique invariant state). On the other hand, if $U$ is not weakly mixing the pure point spectrum of $U$ is nonvoid. Therefore the fixed point algebra $\mathfrak{G}^{\alpha}$ is nontrivial by Proposition 4.1. Let now $F$ be a nonnull function on $L^{2}(X, \mu)$ with $\int F \mathrm{~d} \mu=0$ such that

$$
\underset{N}{\limsup }\left\|\frac{1}{N} \sum_{l=1}^{N} U^{k_{l}} F\right\|>0
$$

for some subsequence $\left\{k_{l}\right\}_{l \in \mathbb{N}}$ of natural numbers of positive lower density (resp. a subsequence of natural numbers). Notice that if $G=G_{1}+i G_{2}$, then $\int|G|^{2} \mathrm{~d} \mu=\int\left(G_{1}^{2}+G_{2}^{2}\right) \mathrm{d} \mu$. This means that (4.4) should be fulfilled at least 
by one of the real or imaginary part of $F$. Thus, we can suppose without loss of generality, that $F$ itself is real. Choose then $f:=F \otimes \xi$ with $\xi \in \oplus_{\lambda \in G} \mathbb{R}^{2}$. Then by (4.2) we have

$$
\underset{N}{\limsup }\left\|\frac{1}{N} \sum_{l=1}^{N} U^{k_{l}} f\right\|_{\mathcal{H}}>0
$$

for the same subsequence $\left\{k_{l}\right\}_{l \in \mathbb{N}}$ of natural numbers of positive lower density (resp. a subsequence of natural numbers) as before. Therefore, we conclude by Proposition 3.5 that if $(X, T, \mu)$ is ergodic but not weakly mixing (resp. weakly mixing but not mixing $),(\mathfrak{G}, \alpha)$ cannot be UWM w.r.t. the fixed point algebra (resp UM).

Theorem 4.3. For the $C^{*}$-dynamical systems considered above, we have that $\mathfrak{G}^{\prime \prime} \cong \pi_{\omega}(\mathfrak{G})^{\prime \prime}$ is a non injective von Neumann factor of type $I I_{1}, I I I_{\lambda}, \lambda \in$ $(0,1)$ or $I I I_{1}$, whenever $G$ is $\{1\},\left\{\lambda^{n}: n=0,1,2, \ldots\right\}$ or $\mathbb{Q}_{+}$respectively, $\pi_{\omega}$ being the GNS representation relative to $\omega$.

Proof. As we are assuming that $L^{2}(X, \mu)$ is infinite dimensional, we have that the positive operator $A$ in (4.2), which is almost periodic in our construction (cf. [27]), has always infinitely many mutually orthogonal eigenvectors corresponding to the eigenvalue 1 . Let $1=\lambda_{1}=\lambda_{2}=\cdots=\lambda_{N}=\cdots$ be an infinite sequence of such eigenvalues. We have $\frac{1}{\sqrt{N}} \sum_{k=1}^{N} \frac{2}{\sqrt{\lambda_{k}}+\sqrt{\lambda_{k}^{-1}}}>4$ whenever $N>16$. Therefore, $\mathfrak{G}^{\prime \prime}$ is not injective by Theorem 2.2 of [16]. On the other hand, by Theorem 3.2 of [16], the centralizer $\left(\mathfrak{G}^{\prime \prime}\right)_{\omega}$ has trivial relative commutant in $\mathfrak{G}^{\prime \prime}$. This implies that $\mathfrak{G}^{\prime \prime}$ is a factor. Finally, Theorem 3.3 of [16] (see also [28]) provides the result relative to the type of the factor $\mathfrak{G}^{\prime \prime}$.

Notice that $\mathfrak{R}^{\prime \prime} \cong \pi_{\omega}(\mathfrak{R})^{\prime \prime}$ is a type $I_{\infty}$ von Neumann factor, see e.g. [11] for the proof.

\section{THE CASE OF $q$-DEFORMED COMMUTATION RELATIONS}

The present section is devoted to show that all the construction can be carried out for the $q$-deformed commutation relations, at least for sufficiently small $q$. For $-1<q<1$, the concrete $C^{*}$-algebras $\mathfrak{R}_{q}$ and its subalgebra $\mathfrak{G}_{q}$ act on the $q$-deformed Fock space $\mathcal{F}_{q}(\mathcal{H})$, which is the completion of the algebraic linear span of the vacuum vector $\Omega$, together with vectors

$$
f_{1} \otimes \cdots \otimes f_{n}, \quad f_{j} \in \mathcal{H}, j=1, \ldots, n, n=1,2, \ldots
$$

w.r.t. the $q$-deformed inner product

$$
\left\langle f_{1} \otimes \cdots \otimes f_{n}, g_{1} \otimes \cdots \otimes g_{m}\right\rangle_{q}:=\delta_{n, m} \sum_{\pi \in \mathbb{P}_{n}} q^{i(\pi)}\left\langle f_{1}, g_{\pi(1)}\right\rangle \cdots\left\langle f_{n}, g_{\pi(n)}\right\rangle,
$$

$\mathbb{P}_{n}$ being the symmetric group of $n$ elements, and $i(\pi)$ the number of inversions of $\pi \in \mathbb{P}_{n}$. The creator $a_{q}^{+}(f)$ is defined as in (2.7), and the corresponding 
annihilator is defined as

$$
\begin{aligned}
a(f) \Omega & =0 \\
a(f)\left(f_{1} \otimes \cdots \otimes f_{n}\right) & =\sum_{k=1}^{n} q^{k-1}\left\langle f_{k}, f\right\rangle f_{1} \otimes \cdots f_{k-1} \otimes f_{k+1} \otimes \cdots \otimes f_{n} .
\end{aligned}
$$

$a_{q}^{+}(f)$ and $a_{q}(f)$ are adjoint each other w.r.t. the inner product (5.1) and satisfy the commutation relations (1.3). The Fock vacuum is defined as $\omega_{q}:=\langle\cdot \Omega, \Omega\rangle$. As for the free situation, a positive contraction $P$ on $\mathcal{H}$ induces a completely positive map $T_{q}(P)$ on the unital $C^{*}$-algebra generated by all the annihilators $a_{q}(f)$ which is an isomorphism, provided $U$ is unitary. The reader is referred to [5] and the literature cited therein, for further details.

Let $\left(\mathfrak{G}_{q}, \alpha_{q}\right)$ be the $C^{*}$-dynamical system where $\mathfrak{G}_{q}$ is the unital $C^{*}$-algebra acting on $\mathcal{F}_{q}(\mathcal{H})$ generated by $\left\{s_{q}(f):=a_{q}(f)+a_{q}^{+}(f) \mid f \in \mathcal{K}_{\mathbb{R}}\right\}$, and $\alpha_{q}\left(s_{q}(f)\right)=$ $s_{q}(U f)$. Here, $\mathcal{K}_{\mathbb{R}}$ is given in (4.1), $\mathcal{H}$ is the completion of $\mathcal{K}_{\mathbb{R}}+i \mathcal{K}_{\mathbb{R}}$ w.r.t. the inner product given in (4.2), and finally $U$ is the unitary acting on $\mathcal{H}$ as described in Section 4.

In order to extend our previous results to the $q$-commutation relations, we need some preparatory results.

Proposition 5.1. Let $\left\{U_{n}\right\}_{n \in \mathbb{N}}, U$ be unitaries acting on $\mathcal{H}$, together with the corresponding Bogoliubov automorphisms $\left\{\alpha_{q}^{(n)}\right\}_{n \in \mathbb{N}} \alpha_{q}$ on $\mathfrak{R}_{q}$, respectively.

If $\lim _{n} U_{n}=U$ in the strong operator topology of $\mathcal{B}(\mathcal{H})$, then $a_{q}^{(n)}$ converges pointwise in norm to $a_{q}$

Proof. It is enough to prove the assertion for each $a_{q}(f), f \in \mathcal{H}$. We get by Remark 1.2 of [5],

$$
\begin{array}{r}
\left\|\alpha^{(n)}\left(a_{q}(f)\right)-\alpha^{(n)}\left(a_{q}(f)\right)\right\|_{\mathcal{B}\left(\mathcal{F}_{q}(\mathcal{H})\right)}=\left\|a_{q}\left(U_{n} f\right)-a_{q}(U f)\right\|_{\mathcal{B}\left(\mathcal{F}_{q}(\mathcal{H})\right)} \\
\leq(1 / \sqrt{1-|q|})\left\|\left(U_{n} f-U f\right)\right\|_{\mathcal{H}}
\end{array}
$$

Let $\mathcal{R}$ be any finite dimensional Hilbert space whose dimension is equal to $d$, together with an orthonormal basis $\left\{e_{j}\right\}_{j=1}^{d}$. It is shown in [20] and [12] that, if $|q|<\sqrt{2}-1$, the unital $C^{*}$-algebra $\mathfrak{R}_{q}$ is isomorphic to $\mathfrak{R}_{0} \equiv \mathfrak{R}$ via a map $\theta$ sending $a_{q}\left(e_{j}\right)$ to $a_{0}\left(e_{j}\right) R$. Here, $R \in \mathfrak{R}_{0}$ is a positive element satisfying

$$
R^{2}=\sum_{j=1}^{d} a_{0}^{+}\left(e_{j}\right) a_{0}\left(e_{j}\right)+\sum_{j, k=1}^{d}\left(a_{0}\left(e_{j}\right) R a_{0}\left(e_{k}\right)\right)^{*}\left(a_{0}\left(e_{k}\right) R a_{0}\left(e_{j}\right)\right) .
$$

Let $M:=\sum_{j=1}^{d} a_{q}^{+}\left(e_{j}\right) a_{q}\left(e_{j}\right)$. It is a positive operator. Furthermore, consider the unitary operator

$$
V=\bigoplus_{m=0}^{\infty} V_{m}: \mathcal{F}_{q}(\mathcal{R}) \rightarrow \mathcal{F}_{0}(\mathcal{R})
$$


defined recursively as

$$
V_{0}:=J_{0}, \quad V_{m}:=\left(J_{1} \otimes V_{m-1}\right) M^{1 / 2}\left\lceil_{\mathcal{R} \otimes m}, \quad m=1,2, \ldots,\right.
$$

where $J_{0} J_{1}$ are the identifications of the (complex multiple of the) vacuum vector and $\mathcal{R}$ in $\mathcal{F}_{q}(\mathcal{R})$, with the corresponding objects in $\mathcal{F}_{0}(\mathcal{R})$, respectively. Then $\rho$ can be written as $R=V M^{1 / 2} V^{*}$.

Lemma 5.2. Let $U$ be a unitary acting on the finite dimensional Hilbert space $\mathcal{R}$. Then $\mathcal{F}_{0}(U) R=R \mathcal{F}_{0}(U)$.

Proof. By taking into account the definition of $M^{1 / 2}, V$ and $R$, it is enough to show that $M$ commutes with $\mathcal{F}_{q}(U)$. We get

$$
\begin{aligned}
& \mathcal{F}_{q}(U) M f_{1} \otimes \cdots \otimes f_{n} \\
= & \sum_{i=1}^{n} q^{i-1}\left(\sum_{j=1}^{d}\left\langle f_{i}, e_{j}\right\rangle U e_{j}\right) \otimes U f_{1} \otimes \cdots \otimes U f_{i-1} \otimes U f_{i+1} \otimes \cdots \otimes U f_{n} \\
= & \sum_{i=1}^{n} q^{i-1}\left(\sum_{j=1}^{d}\left\langle U f_{i}, U e_{j}\right\rangle U e_{j}\right) \otimes U f_{1} \otimes \cdots \otimes U f_{i-1} \otimes U f_{i+1} \otimes \cdots \otimes U f_{n} \\
= & \sum_{i=1}^{n} q^{i-1} U f_{i} \otimes U f_{1} \otimes \cdots \otimes U f_{i-1} \otimes U f_{i+1} \otimes \cdots \otimes U f_{n} \\
= & M \mathcal{F}_{q}(U) f_{1} \otimes \cdots \otimes f_{n} .
\end{aligned}
$$

In addition, it is shown in [16], Section 5, that the previous result extends to the case of any separable Hilbert space $\mathcal{R}$, where $\theta$ is the inductive limit of the corresponding isomorphisms $\theta_{n}$ for each increasing sequence of $d_{n}$-dimensional subspaces $\mathcal{R}_{n}$ such that $\bigcup_{n} \mathcal{R}_{n}$ is dense in $\mathcal{R}$. We refer the reader to the above mentioned paper [16], for further details relative to the isomorphism $\theta$ realizing the equivalence between $\mathfrak{R}_{q}$ and $\mathfrak{R}_{0}$, when $|q|<\sqrt{2}-1$ (known in the literature as $E_{q}(\mathcal{R})$ and $E_{0}(\mathcal{R})$, respectively).

Theorem 5.3. There exists an isomorphism $\theta: \mathfrak{R}_{q} \rightarrow \mathfrak{R}_{0}$ which intertwines any Bogoliubov automorphism: $\theta \circ \alpha_{q}=\alpha_{0} \circ \theta$, provided $|q|<\sqrt{2}-1$.

Proof. Let $U$ be the unitary acting on $\mathcal{H}$ generating the Bogoliubov automorphism on the algebras $\mathfrak{R}_{0}$ and $\mathfrak{R}_{q},|q|<\sqrt{2}-1$. Let $K$ be the Cayley transform of $U$, together with the resolution of the identity $\lambda \mapsto E(\lambda)$ of $K$, which is supposed to be right-continuous (in the strong operator topology). Define the saw-tooth function

$$
h(\lambda):=\lambda-2 k \pi, \quad \lambda \in(2 k \pi, 2(k+1) \pi], \quad k \in \mathbb{Z} .
$$

It is easy to show that $H:=\int \lambda \mathrm{d} E(\lambda)$ is a bounded selfadjoint operator such that $U=e^{i H}$. Fix an increasing sequence $\left\{\mathcal{H}_{n}\right\}$ of finite dimensional subspaces 
such that $\bigcup_{n} \mathcal{H}_{n}$ is dense in $\mathcal{H}$, togeheter with the associated selfadjoint projections $P_{n}$. Define $U_{n}:=e^{i P_{n} H P_{n}}$. We easily get $\lim _{n} U_{n}=U$ in the strong topology of $\mathcal{R}$. There exists an isomorphism $\theta: \mathfrak{R}_{q} \rightarrow \mathfrak{R}_{0}$ intertwining $\alpha_{p}^{(n)}$ and $\alpha_{0}^{(n)}$. This can be done by considering for each fixed $n$, the sequence of unitary operators $U_{n}\left\lceil\mathcal{H}_{m}, m>n\right.$. Lemma 5.6 of [16] leads to the claim by taking into account Lemma 5.2. Fix $A \in \mathfrak{R}_{q}$. By Proposition 5.1 we get

$$
\theta\left(\alpha_{q}(A)\right)=\theta\left(\lim _{n} \alpha_{q}^{(n)}(A)\right)=\lim _{n} \theta\left(\alpha_{q}^{(n)}(A)\right) \lim _{n} \alpha_{0}^{(n)}(\theta(A))=\alpha_{0}(\theta(A)),
$$

which is the assertion.

Theorem 5.4. For the $C^{*}$-dynamical system $\left(\mathfrak{G}_{q}, \alpha_{q}\right)$, all the assertions of Theorem 4.2 hold true, provided $|q|<\sqrt{2}-1$.

Proof. Fix the $C^{*}$-dynamical system $\left(\mathfrak{R}_{q}, \alpha_{q}\right)$. By Theorem 5.3. It is conjugate to the $C^{*}$-dynamical system $\left(\mathfrak{R}_{0}, \alpha_{0}\right)$, where $\alpha_{q}, \alpha_{0}$ are Bogoliubov automorphisms generated on $\mathfrak{R}_{q}, \mathfrak{R}_{0}$ by the same orthogonal operator. In addition, the isomorphism described in Proposition 5.3 intertwines the corresponding Fock vacua $\omega_{q}$ and $\omega_{0} \cdot{ }^{5}$ By taking into account of Proposition 3.1 and (4.3), $\left(\mathfrak{R}_{q}, \alpha_{q}\right)$ is UE w.r.t. the fixed point subalgebra (resp UWM or UM), provided $U$ is ergodic (resp. weakly mixing or mixing). Then $\left(\mathfrak{G}_{q}, \alpha_{q}\right)$ is by restriction, UE w.r.t. the fixed point subalgebra, UWM or UM, provided $U$ is ergodic, weakly mixing or mixing, respectively. On the other hand, suppose that the classical ststem $(X, T, \mu)$ is ergodic (resp. weakly mixing) but not weakly mixing (resp. mixing). We can choose as in the proof of Theorem $4.2 f \in \mathcal{K}_{\mathbb{R}}$ such that

$$
\underset{N}{\limsup }\left\|\frac{1}{N} \sum_{l=1}^{N} U^{k_{l}} f\right\|_{\mathcal{H}}>0
$$

for some subsequence $\left\{k_{l}\right\}_{l \in \mathbb{N}}$ of natural numbers of positive lower density (resp. a subsequence of natural numbers). Then we have

$$
\begin{gathered}
0=\lim _{N} \frac{1}{N}\left\|\sum_{k=1}^{N} \alpha_{q}^{k}\left(s_{q}(f)\right) \Omega\right\|=\lim _{N}\left\|\frac{1}{N} \sum_{k=1}^{N} \alpha_{q}^{k}\left(a_{q}^{+}(f)\right) \Omega\right\| \\
=\limsup _{N}\left\|\frac{1}{N} \sum_{l=1}^{N} U^{k_{l}} f\right\|>0
\end{gathered}
$$

which is a contradiction. Thus, $\left(\mathfrak{G}_{q}, \alpha_{q}\right)$ cannot be UWM w.r.t. the fixed point subalgebra (resp. UM). Finally, in the case when $(X, T, \mu)$ is weakly mixing or mixing, the fixed point algebra of $\left(\mathfrak{R}_{q}, \alpha_{q}\right)$ is trivial. Then by restriction, the fixed point algebra of $\left(\mathfrak{G}_{q}, \alpha_{q}\right)$ is trivial as well. In the ergodic case, the fixed point of $\left(\mathfrak{G}_{q}, \alpha_{q}\right)$ is non trivial by Proposition 4.1.

\footnotetext{
${ }^{5}$ Notice that it is unclear if such isomorphism sends $\mathfrak{G}_{q}$ onto $\mathfrak{G}_{0}$. Thus, it is unclear if $\left(\mathfrak{G}_{q}, \alpha_{q}\right)$ is conjugate to $\left(\mathfrak{G}_{0}, \alpha_{0}\right)$.
} 
Consider now the $q$-shift. Namely, let $u: \ell_{\mathbb{R}}^{2}(\mathbb{Z}) \mapsto \ell_{\mathbb{R}}^{2}(\mathbb{Z})$ be the shift acting on $\ell_{\mathbb{R}}^{2}(\mathbb{Z})$, and

$$
\mathcal{K}_{\mathbb{R}}:=\ell_{\mathbb{R}}^{2}(\mathbb{Z}) \bigotimes\left(\oplus_{\lambda \in G} \mathbb{R}^{2}\right)
$$

$\mathcal{H}$ will be the completion of $\mathcal{K}_{\mathbb{R}}+i \mathcal{K}_{\mathbb{R}}$ w.r.t. the inner product given in (4.2). Define $U$ and $V(t)$ as in Section 4, and finally $\mathfrak{G}_{q}$ as at the beginning of the present section. In this case (as well as in all the cases previously described in the present section for each $-1<q<1) \mathfrak{G}_{q}^{\prime \prime}$ is non injective von Neumann factor of type $I I_{1}, I I I_{\lambda}, \lambda \in(0,1)$ or $I I I_{1}$, whenever $G$ is $\{1\},\left\{\lambda^{n}: n=\right.$ $0,1,2, \ldots\}$ or $\mathbb{Q}_{+}$respectively.

In [11], it was proven for each $-1<q<1$, that the $q$-shift is UM in the case when the modular theory is trivial (i.e. when $G=\{1\}$ in (4.1)). The same proof of Theorem 3 of [11] allows us to extend the previous results to all cases $-1<q<1$, at least in the case of the shift. We have then proven the following

Proposition 5.5. For each $-1<q<1$, the $C^{*}$-dynamical system $\left(\mathfrak{G}_{q}, \alpha_{q}\right)$ ( $\alpha_{q}$ being the $q$-shift) is UM, with the Fock vacuum $\omega_{q}$ the unique invariant state.

We can conjecture that all the results described in the present section for the $C^{*}$-dynamical systems based on the $q$-commutation relations, can be extended to all $q \in(0,1)$. Unfortunately, it is not known if all the $C^{*}$-algebras $\mathfrak{R}_{q}$ are isomorphic for any $q \in(-1,1)$. In addition, an estimation similar to Proposition 3.1 and to Proposition 2 of [11] is not yet available for the general case when $q \neq 0$ and the involved Bogoliubov automorphism is not the shift.

\section{ACKNOWLEDGEMENT}

The second-named author (F. M.) thanks the MOHE grant FRGS0308-91.

\section{REFERENCES}

[1] Abadie B., Dykema K. Unique ergodicity of free shifts and some other automorphisms of $C^{*}$-algebras, J. Operator Theory, to appear.

[2] Accardi L., Mukhamedov F. A note on noncommutative unique ergodicity and weighted means, Linear Alg. Appl. 430 (2009), 782-790.

[3] Albeverio S., Høegh-Krohn R. Frobenius theory for positive maps of von Neumann algebras, Commun. Math. Phys. 64 (1978), 83-94.

[4] Bezuglyi S. I., Golodets, V. Ya. Dynamical entropy for Bogoliubov actions of free abelian groups on the CAR-algebra, Ergodic Theory Dynam. Sys., 17 (1997), 757782.

[5] Bożejko M., Kummerer B., Speicher R. q-gaussian processes: non-commutative and classical aspects, Commun. Math. Phys. 185 (1997), 129-154.

[6] Bratteli O., Robinson D.W., Operator algebras and quantum statistical mechanics II, Springer, New York-Heidelberg-Berlin, 1981.

[7] Brown N. P., Choda, M. Approximation entropies in crossed products with an application to free shifts, Pacific J. Math. 198 (2001), 331-346.

[8] Brown N. P., Dykema K., Shlyakhtenko, D. Topological entropy of free product automorphisms, Acta Math. 189 (2002), 1-35. 
[9] Damanik D. Strictly ergodic subshifts and associated operators, Spectral theory and mathematical physics: a Festschrift in honor of Barry Simon's 60th birthday, 505538, Proc. Sympos. Pure Math 76, Providence, RI, 2007.

[10] Dykema K. Topological entropy of some automorphisms of reduced amalgamated free product $C^{*}$-algebras, Ergodic Theory Dynam. Sys. 21 (2001), 1683-1693.

[11] Dykema K., Fidaleo F. Unique mixing of the shift on the $C^{*}$-algebras generated by the q-canonical commutation relations, Houston J. Math., to appear.

[12] Dykema K., Nica A. On the Fock representation of the q-commutation relations, J. Reine Angew. Math. 440 (1993), 201-212.

[13] Fidaleo F. On strong ergodic properties of quantum dynamical systems, Infin. Dimens. Anal. Quantum Probab. Relat. Top., to appear.

[14] Fidaleo F., Mukhamedov F. Strict weak mixing of some $C^{*}$-dynamical systems based on free shifts, J. Math. Anal. Appl. 336 (2007), 180-187.

[15] Golodets V. Ya., Neshveyev S. V. Dynamical entropy for Bogoliubov actions of torsion-free abelian groups on the CAR-algebra, Ergodic Theory Dynam. Sys., 20 (2000), 1111-1125.

[16] Hiai F. q-deformed Araki-Woods algebras, in: Constanca proceedings 2003, 169-202 Theta, Bucharest.

[17] Jajte R., Strong limit theorems in non-commutative probability, Lecture Notes in Math. 1110, Springer, New York-Heidelberg-Berlin, 1984.

[18] Jewett R. I. The pervalence of uniquely ergodic systems, J. Math. Mec. 19 (1970), $717-729$.

[19] Jones K. L., Lin M. Ergodic theorems of weak mixing type, Proc. Amer. Math. Soc. $\mathbf{5 7}(1976), 50-52$.

[20] Jorgensen P. E. T., Schmitt L. M., Werner R. F. q-canonical commutation relations and stability of Cuntz algebra, Pacific J. Math. 165 (1994), 131-151.

[21] Kornfeld I. P., Sinai Ya. G., Fomin S. V., Ergodic Theory, Springer, Springer, New York-Heidelberg-Berlin, 1982.

[22] Krieger W. On unique ergodicity, in "Proceedings of the Sixth Berkeley Symposium on Mathematical Statistics and Probability (1970/1971), Vol. II: Probability theory". Univ. California Press, Berkeley, 1972, 327-346.

[23] Lin M. Mixing for Markov operators, Z. Wahrscheinlichkeitstheor. 19 (1971), 231242.

[24] Mukhamedov F. On strictly weakly mixing $C^{*}$-dynamical systems, Funct. Anal. Appl. 27(2007), 311-313.

[25] Mukhamedov F., Temir S. A few remarks on mixing properties of $C^{*}$-dynamical systems, Rocky Mount. J. Math. 37 (2007), 1685-1703.

[26] Neshveyev S., Stormer E. Dynamical Entropy in Operator Algebras, Springer, New York-Heidelberg-Berlin, 2006.

[27] Niculescu C., Ströh A., Zsidó L., Noncommutative extensions of classical and multiple recurrence theorems, J.Operator Theory, 50 (2003), 3-52.

[28] Shlyakhtenko D., Free quasi-free states, Pacific J. Math. 177 (1997), 329-368.

[29] Stomer E., Voiculescu D. Entropy of Bogoliubov automorphisms of the canonical anticommutation relations, Commun. Math. Phys. 133 (1990), 521-542.

[30] Strătilă S. Modular theory in operator algebras, Abacus press, Tunbridge Wells, Kent 1981.

[31] Strătilă S., Zsidó L. Lectures on von Neumann algebras, Abacus press, Tunbridge Wells, Kent 1979.

[32] Voiculescu D., Dynamical approximation entropies and topological entropy in operator algebras, Commun. Math. Phys. 170 (1995), 249-281.

[33] Voiculescu D., Dykema K., Nica A. Free random variables, CRM monograph serie Vol. 1, AMS, 1992. 
Francesco Fidaleo, Department of Comput. \& Theor. Sci., Faculty of Science, IIUM, P.O. Box, 141, 25710, Kuantan, Pahang, Malaysia. Permanent address: Dipartimento di Matematica, II Università di Roma "Tor Vergata", Via della Ricerca Scientifica, 00133 Roma, Italia

E-mail address: fidaleo@mat.uniroma2.it

Farrukh Mukhamedov, Department of Comput. \& Theor. Sci., Faculty of Science, IIUM, P.O. Box, 141, 25710, Kuantan, Pahang, Malaysia

E-mail address: far75m@yandex.ru, farrukh_m@iiu.edu.my 